\title{
Content based Image Retrieval using Sectorisation of Self Mutated Hybrid Wavelet Transforms
}

\author{
Yogita D. Shinde \\ M.E Student, Dept. of Computer Engineering \\ Pimpri Chinchwad College of Engg. \\ Akurdi, Pune, India
}

\author{
Sudeep D. Thepade \\ Professor, Dept. of Computer Engineering \\ Pimpri Chinchwad College of Engg. \\ Akurdi, Pune, India
}

\begin{abstract}
Content based image retrieval (CBIR) is an image retrieval process which involves mainly extraction of features based on contents of an image which uniquely identifies an image from other images in the database. Hybrid Wavelet Transform is formed using two orthogonal transforms. In this paper, Self Mutated Hybrid Wavelet transform (SMHWT) is used which is formed by using same component transform. In Proposed algorithm, feature extraction is done by applying sectorisation on Self Mutated Hybrid Wavelet transformed images. To test the performance of the proposed method, total 1000 queries were fired on the image database containing 1000 images of 10 categories. Manhattan Distance is used for similarity measurement. Performances proposed algorithm is evaluated using average precision. Results show that the proposed Self Mutated Hybrid Wavelet Transform containing Sine transform as a component gives better performance improvement across all tried variations of SMHWTs.
\end{abstract}

\section{Keywords}

CBIR; Self Mutation; Hybrid Wavelet Transform; Sectorisation; Manhattan Distance

\section{INTRODUCTION}

Day by day, there is an explosive growth in the internet, data storage capacity, digital imaging devices which motivates the people to communicate and express themselves by sharing images and videos. This results into huge amount of digital image information on internet. To retrieve particular image from this large database is very challenging task. Traditionally there was Text based image retrieval. But this method involves manual annotation and hence having major drawbacks of being subjective, complex, time consuming and depends on human so one cannot automate this process [1].

Content Based Image Retrieval (CBIR) is an image retrieval process where images are retrieved by its content like color, shape, texture, transformed image content. It automates the process of image retrieval and having many applications in Trademark registration, security, and online photo sharing [2].

\section{LITERATURE SURVEY}

Various techniques have been developed for CBIR based on spatial contents and/or transformed image contents. Techniques like Color Histogram, Color averaging [3], Color Correlogram [4] and Block Truncation Coding [5] etc. are used for extracting color feature extraction but color feature based image retrieval gets affected due to illumination differences among images. Gradient operator with slope magnitude method [6], Morphological operator [7] can be used for shape feature extraction. Shape based image retrieval are having drawback of not being rotation invariant and need same sized images in the database.
Orthogonal transforms have energy compaction property which can be used to reduce the size of feature vector in CBIR [8]. Also fractional energy of various transformed images using various orthogonal transforms like DCT, Walsh, Haar and Kekre transforms is taken for image retrieval [9]. Features extracted using sectorisation of transformed images is free from limitations like rotation variance and color illumination differences and needs same sized images in database. Hybrid wavelet transform sectorisation gives better results than individual orthogonal transforms [10]. In this paper, sectorisation of Self Mutated Hybrid Wavelet transform (SMHWT) is considered for feature extraction. Here total 7 orthogonal transform is considered for generation of SMHWT like Sine, Cosine, Haar, Walsh, Kekre, Slant, Hartley, etc.

\section{SECTORISATION OF}

\section{TRANSFORMED IMAGES}

Sectorisation of transformed image can be done using following steps [10] as shown in following Figure 1.

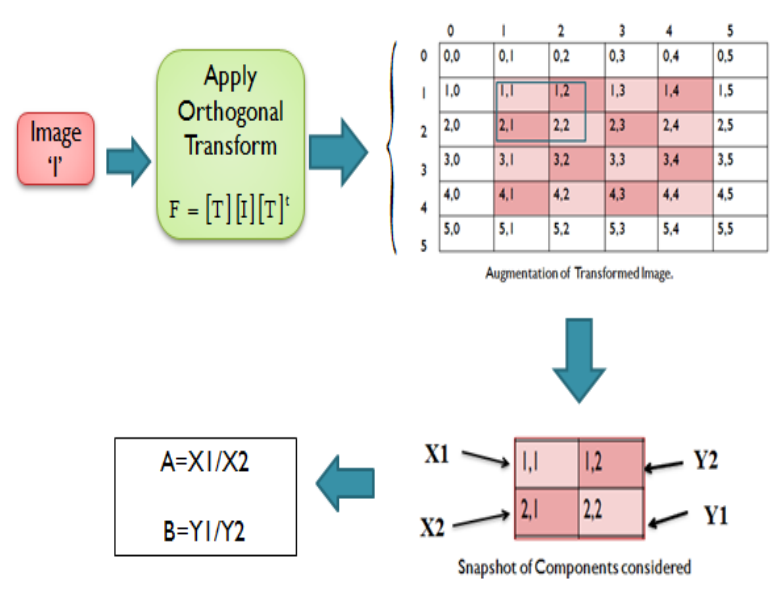

Fig 1: Sectorisation of transformed image.

Modify an image by ignoring first row, column and last row column to get an Augmented Transformed Image. Divide the transformed image into non overlapping window of size $2 \times 2$. For each window, consider values of variable X1, Y1, X2, Y2 as shown in the following fig. 1. Calculate value of $\mathrm{A}=\mathrm{X} 1 / \mathrm{X} 2$, $\mathrm{B}=\mathrm{Y} 1 / \mathrm{Y} 2$.

Depending on sign of 'A 'and 'B', sector number of respective window is determined using conditions given in table 1 . With table 1 four sectors are formed. With four sectors, eight sectors can be obtained using conditions given in table 2 . With four sectors, 12 sectors can be obtained using conditions given in 
table 3. With four sectors, sixteen sectors can be obtained using conditions given in table 4 .

Table 1. Formation of Four Sectors

\begin{tabular}{|c|c|c|}
\hline $\begin{array}{c}\text { Sign } \\
\text { of A }\end{array}$ & $\begin{array}{c}\text { Sign } \\
\text { of B }\end{array}$ & $\begin{array}{c}\text { Sector } \\
\text { Number }\end{array}$ \\
\hline+ & + & 1 \\
\hline+ & - & 2 \\
\hline- & - & 3 \\
\hline- & + & 4 \\
\hline
\end{tabular}

Table 2. Formation of Eight Sectors

\begin{tabular}{|c|c|}
\hline Sector Number & Condition \\
\hline $1,4,5,8$ & $|\mathrm{~A}|>=|\mathrm{B}|$ \\
\hline $2,3,6,7$ & $|\mathrm{~B}|>=|\mathrm{A}|$ \\
\hline
\end{tabular}

Table 3. Formation of twelve sectors

\begin{tabular}{|l|l|}
\hline $\begin{array}{l}\text { Sector } \\
\text { Number }\end{array}$ & Condition \\
\hline $1,4,7,10$ & $|A|>=\sqrt{3} *|B|$ \\
\hline $2,5,8,11$ & $|1 / \sqrt{3} *| A|<=B|<=\sqrt{3} *|A|$ \\
\hline $3,6,9,12$ & Otherwise \\
\hline
\end{tabular}

Table 4. Formation of Sixteen sectors

\begin{tabular}{|l|l|}
\hline Sector Number & Condition \\
\hline $1,5,9,13$ & $|A| \geq 2 *|B|$ \\
\hline $2,6,10,14$ & $|A| \leq|B| \leq 4 *|A|$ \\
\hline $3,7,11,15$ & $|B| \geq 2 *|A|$ \\
\hline $4,8,12,16$ & Otherwise \\
\hline
\end{tabular}

\section{SELF MUTATED HYBRID} WAVELET TRANSFORM

Self Mutated Hybrid Wavelet Transform (say SM-A) of size $\mathrm{N}^{2} \times \mathrm{N}^{2}$ is formed by orthogonal transform (say A) of size $\mathrm{N} \times \mathrm{N}$ as shown in following equation (1). Here first N rows of SMA are formed by calculating the product of each element of first row with each column of A. second row of $\mathrm{A}$ is appended by zeros till size becomes $\mathrm{N}^{2}$ and then shift rotate this row to get next N rows of SM-A. Similarly other rows of SM-A are formed as shown in following fig. 2. In this paper 7 orthogonal transforms like Sine, Cosine, Haar, Walsh, Kekre, Slant, and Hartley etc. are used for generation of different combinations of Self Mutated Hybrid Wavelet Transforms.

$$
A=\left[\begin{array}{cccc}
a_{11} & a_{12} & \cdots & a_{1 N} \\
a_{21} & a_{21} & \ldots & a_{2 N} \\
\vdots & \vdots & \vdots & \vdots \\
a_{N 1} & a_{N 2} & \cdots & a_{N N}
\end{array}\right]
$$

\begin{tabular}{|c|c|c|c|c|c|c|c|c|c|c|c|c|}
\hline$a_{1 !}\left[\begin{array}{c}a_{n 1} \\
a_{n !} \\
\vdots \\
a_{n n}\end{array}\right]$ & $a_{12}\left[\begin{array}{c}a_{n} \\
a_{21} \\
\vdots \\
a_{n n}\end{array}\right]$ & $\ldots$ & $a_{125}\left[\begin{array}{c}a_{n} \\
a_{n: 1} \\
\vdots \\
a_{n n}\end{array}\right]$ & $a_{1 ! 1}\left[\begin{array}{c}a_{1:} \\
a_{n:} \\
\vdots \\
a_{n: 1}\end{array}\right]$ & {$\left[\begin{array}{c}a_{1:} \\
a_{12} \\
a_{2:} \\
\vdots \\
a_{v: 3}\end{array}\right]$} & '. & $a_{1: N}\left[\begin{array}{c}a_{n} \\
a_{n:} \\
\vdots \\
a_{n:}\end{array}\right]$ & $\cdots$ & $a_{11}\left[\begin{array}{c}a_{12 x} \\
a_{2 x} \\
\vdots \\
a_{x x}\end{array}\right]$ & $a_{12}\left[\begin{array}{c}a_{12} \\
a_{2 x} \\
\vdots \\
a_{x i x}\end{array}\right]$ & .'. & 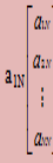 \\
\hline$a_{21}$ & $a_{22}$ & $\ldots$ & $a_{2 N}$ & 0 & 0 & $\ldots$ & 0 & 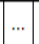 & 0 & 0 & \begin{tabular}{|l|}
$\ldots$ \\
\end{tabular} & 0 \\
\hline 0 & 0 & $\ldots$ & 0 & $a_{21}$ & $a_{22}$ & $\ldots$ & $a_{2 \mathrm{~N}}$ & $\ldots$ & 0 & 0 & $\ldots$ & 0 \\
\hline$\vdots$ & $\vdots$ & $\vdots$ & $\vdots$ & $\vdots$ & $\vdots$ & $\vdots$ & $\vdots$ & \begin{tabular}{|l|}
$\ldots$ \\
\end{tabular} & $\vdots$ & $\vdots$ & $\vdots$ & $\vdots$ \\
\hline 0 & 0 & 0 & 0 & 0 & 0 & 0 & 0 & $\ldots$ & $a_{21}$ & $a_{22}$ & $\ldots$ & $a_{2 N}$ \\
\hline$a_{31}$ & $a_{32}$ & $\ldots$ & $a_{33}$ & 0 & 0 & $\ldots$ & 0 & $\ldots$ & 0 & 0 & $\ldots$ & 0 \\
\hline 0 & 0 & $\ldots$ & 0 & $a_{31}$ & $a_{32}$ & $\ldots$ & $a_{33} \mathrm{~N}$ & \begin{tabular}{|l|}
$\ldots$ \\
\end{tabular} & 0 & 0 & \begin{tabular}{|l|}
$\ldots$ \\
\end{tabular} & 0 \\
\hline$\vdots$ & $\vdots$ & $\vdots$ & $\vdots$ & $\vdots$ & $\vdots$ & $\vdots$ & $\vdots$ & \begin{tabular}{|l|}
$\ldots$ \\
\end{tabular} & $\vdots$ & $\vdots$ & \begin{tabular}{|l|l|}
$\vdots$ \\
\end{tabular} & $\vdots$ \\
\hline 0 & 0 & $\ldots$ & 0 & 0 & 0 & $\ldots$ & 0 & $\ldots$ & $a_{31}$ & $a_{32}$ & \begin{tabular}{|l|}
$\ldots$ \\
\end{tabular} & $a_{3 N}$ \\
\hline$\vdots$ & $\vdots$ & $\vdots$ & $\vdots$ & $\vdots$ & $\vdots$ & $\vdots$ & $\vdots$ & \begin{tabular}{|l|}
.. \\
\end{tabular} & $\vdots$ & $\vdots$ & \begin{tabular}{|l|l|}
$\vdots$ \\
\end{tabular} & $\vdots$ \\
\hline $2 \mathrm{NI}$ & $a \times 2$ & $\ldots$ & $2 \mathrm{~N}$ & 0 & 0 & $\ldots$ & 0 & $\ldots$ & 0 & 0 & $\ldots$ & 0 \\
\hline 0 & 0 & $\ldots$ & 0 & $2 \mathrm{NI}$ & $a \times 2$ & $\ldots$ & $a N$ & $\ldots$ & 0 & 0 & $\ldots$ & 0 \\
\hline$\vdots$ & $\vdots$ & $\vdots$ & $\vdots$ & $\vdots$ & $\vdots$ & $\vdots$ & $\vdots$ & \begin{tabular}{|l|}
$\ldots$ \\
\end{tabular} & $\vdots$ & $\vdots$ & \begin{tabular}{|l|l|}
$\vdots$ \\
\end{tabular} & $\vdots$ \\
\hline 0 & 0 & $\ldots$ & 0 & 0 & 0 & $\ldots$ & 0 & \begin{tabular}{|l|}
... \\
\end{tabular} & an: & $2 \mathrm{~N} 2$ & $\ldots$ & an \\
\hline
\end{tabular}

Fig 2: Generation of SMHWT

\section{PROPOSED CBIR TECHNIQUE}

In proposed algorithm, unique Features of an image were extracted using sectorisation of Self Mutated Hybrid Wavelet Transformed images. Orthogonal transforms like Sine, Cosine, Haar, Walsh, Slant, Kekre, Hartley are used to form SMHWT. Features of Query image and Features of images in database are compared using Manhattan Distance [11] given by following equation (2), here $f q$ be the feature vector of the query image and $f d$ be the feature vector of images in database.

$$
S=\sum_{j=1}^{n}|f q(j)-f d(j)|
$$

\section{EXPERIMENTATION ENVIRONMENT}

The Wang image dataset [12] used for experimentation is consists of 10 different categories and 100 images per each image category. Hence, total 1000 images collection is there. MATLAB with support of Windows environment is used for experimentation. 


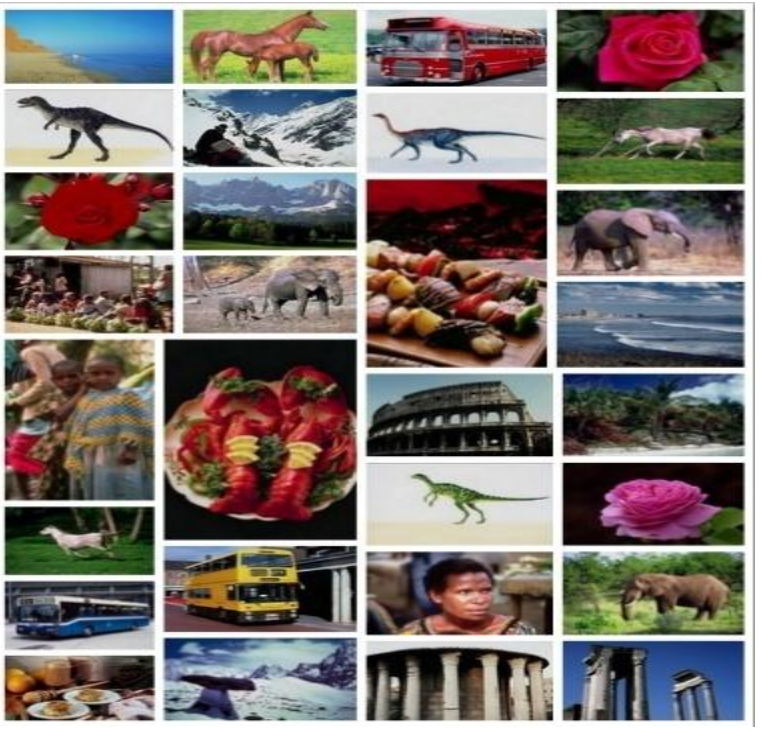

Fig 3: Sample Images in database

\section{RESULTS AND DISCUSSION}

To test the performance of proposed algorithm, feature extraction is done with sectorisation of individual orthogonal transformed images and with the self mutated hybrid wavelet transformed images. In proposed technique seven SMHWTs such as SM-Sine, SM-Cosine, SM-Haar, SM-Walsh, SMKekre, SM-Slant, SM-Hartley are used and its performance is used. Total 1000 queries were fired on database and average precision is used to evaluate the performance of proposed technique.

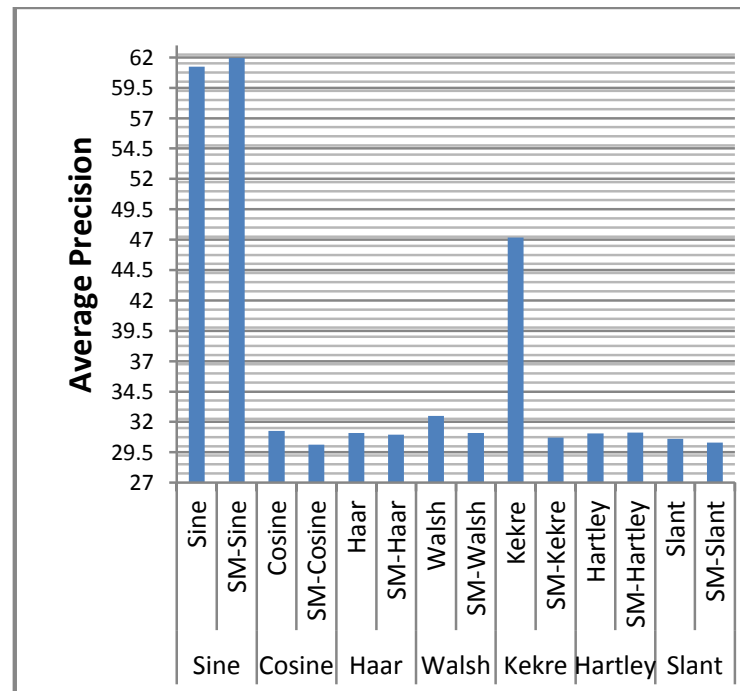

Transforms Used

Fig 4: Results of proposed method with different SMHWTs

Figure 4 gives performance comparison of individual orthogonal transform and corresponding SMHWTs. In this best average precision is observed by SM-Sine, Sine followed by Kekre Transform.
Table 5. Results of proposed algorithm with different combination of Self Mutated Hybrid Transforms

\begin{tabular}{|c|c|c|c|c|c|c|}
\hline \multirow{2}{*}{\multicolumn{2}{|c|}{$\begin{array}{l}\text { Transform } \\
\text { Used }\end{array}$}} & \multicolumn{4}{|c|}{$\begin{array}{l}\text { Average Precision with } \\
\text { Number of Sectors }\end{array}$} & \multirow{3}{*}{$\begin{array}{l}\text { Average } \\
61.23\end{array}$} \\
\hline & & \multirow{2}{*}{$\begin{array}{l}4 \\
62.56\end{array}$} & \multirow{2}{*}{$\begin{array}{l}8 \\
61.96\end{array}$} & \multirow{2}{*}{$\begin{array}{l}12 \\
60.28\end{array}$} & \multirow{2}{*}{$\begin{array}{l}16 \\
60.12\end{array}$} & \\
\hline \multirow{2}{*}{ Sine } & Sine & & & & & \\
\hline & $\begin{array}{l}\text { SM- } \\
\text { Sine }\end{array}$ & 61.84 & 62.52 & 62.94 & 60.5 & 61.95 \\
\hline \multirow{2}{*}{ Cosine } & Cosine & 33.62 & 32.22 & 29.68 & 29.5 & 31.255 \\
\hline & $\begin{array}{l}\text { SM- } \\
\text { Cosine }\end{array}$ & 33.18 & 30.12 & 29.12 & 28.12 & 30.135 \\
\hline \multirow{2}{*}{ Haar } & Haar & 33.42 & 30.9 & 30.7 & 29.38 & 31.1 \\
\hline & $\begin{array}{l}\text { SM- } \\
\text { Haar }\end{array}$ & 32.78 & 31.26 & 30.48 & 29.22 & 30.935 \\
\hline \multirow{2}{*}{ Walsh } & Walsh & 35.84 & 33.22 & 30.94 & 29.92 & 32.48 \\
\hline & $\begin{array}{l}\text { SM- } \\
\text { Walsh }\end{array}$ & 33.14 & 31.28 & 30.18 & 29.76 & 31.09 \\
\hline \multirow{2}{*}{ Kekre } & Kekre & 46.34 & 47.5 & 48.42 & 46.42 & 47.17 \\
\hline & $\begin{array}{l}\text { SM- } \\
\text { Kekre }\end{array}$ & 32.54 & 31.62 & 30.15 & 28.55 & 30.715 \\
\hline \multirow[b]{2}{*}{ Hartley } & Hartley & 32.9 & 31.66 & 30.32 & 29.32 & 31.05 \\
\hline & $\begin{array}{l}\text { SM- } \\
\text { Hartley }\end{array}$ & 32.96 & 31.04 & 30.89 & 29.56 & $\begin{array}{l}31.112 \\
5\end{array}$ \\
\hline \multirow{2}{*}{ Slant } & Slant & 32.22 & 30.94 & 30.08 & 29.18 & 30.605 \\
\hline & $\begin{array}{l}\text { SM- } \\
\text { Slant }\end{array}$ & 31.96 & 31.72 & 29.3 & 28.2 & 30.295 \\
\hline
\end{tabular}

Table 5 gives the results of CBIR using sectorisation of Self Mutated Hybrid wavelet transformed images. Here SM-Sine transform with 12 sectors gives better average precision than other considered SMHWTs. Performance of proposed algorithm increases with increase in number of sectors (up to 12 sectors) used in feature extraction in case of SM-Sine transform. Excluding SM-Sine Transform, for all other transform better performance is observed with 4 sectors used in feature extraction.

\section{CONCLUSION}

Proposed CBIR algorithm is based on Transform Sectorisation technique and hence it is free from limitations like illumination differentiations among images, rotation variance, needing same sized images used in database. In this total seven variation of Self Mutated Hybrid Wavelet Transform is considered for experimentation. 
Proposed CBIR technique involves Sectorisation of Self Mutated Hybrid Wavelet Transformed Images for feature extraction. Among all tried combinations, proposed technique gives better performance with SM-Sine transform with 12 sectors used in feature extraction. Performance of proposed algorithm decreases with increase in number of sectors used in feature extraction except SM-Sine Transform. Performance comparison shows that proposed algorithm performs better with only SM-Sine transform because for other proposed variations performance is decreased.

\section{REFERENCES}

[1] Sudeep D. Thepade, Yogita D. Shinde, "Improvisation of Content Based Image retrieval using Color Edge Detection with various Gradient Filters and Slope Magnitude Method" International Conference on Computing, Communication, Control and Automation (ICCUBEA-2015), 26 -27 Feb 2015, India.

[2] Muller H., Michoux N., Bandon D., and Geissbuhler A., "A Review of Content Based Image Retrieval Systems In Medical Applications-Clinical Benefits and Future Directions", International Journal Medical Inf., Volume 73, Number 1, pp.1-23, 2004

[3] Akshay Maloo, Sudeep D Thepade and Dr. H.B.Kekre, "CBIR Feature Vector Dimension Reduction with Eigenvectors of Covariance Matrix using Row, Column and Diagonal Mean Sequences", International Journal of Computer Applications 3(12):39-46, July 2010.

[4] B V Patel and B B Meshram, "Content Based Video Retrieval Systems", In 21st International Conference on Pattern Recognition (ICPR2012) November 11-15, 2012. Tsukuba, Japan.

[5] Dr.H.B.Kekre, Sudeep D. Thepade, "Image Retrieval using Augmented Block Truncation Coding Techniques", International Conference on Advances in
Computing, Communication and Control (ICAC3'09), January 23-24, 2009, India.

[6] Dr. H. B. Kekre, Priyadarshini Mukherjee, Shobhit Wadhwa," Image Retrieval with shape features extracted using gradient operators ans slope magnitude method with BTC", International Journal of Computer Applications (0975 - 8887), Volume 6- No.8, September 2010.

[7] Rafael C. Gonzalez, Richard E. Woods, "Digital Image Processing", second edition, Pearson publication, Inc. 2005.

[8] Dr. H. B. Kekre, Sudeep D. Thepade, Archana Athawale, Anant Shah, Prathamesh Verlekar, Suraj Shirke," Performance Evaluation Of Image Retrieval Using Energy Compaction And Image tiling Over Det Row Mean And Dct Column Mean ”,Thinkquest,2010-2011, pp 158-167.

[9] Dr. H. B. Kekre, Dr. Sudeep D. Thepade,Varun K. Banura, Ankit Khandelwal," Augmentation of Image Retrieval using Fractional Coefficients of Hybrid Wavelet Transformed Images with Seven Image Transforms", IJCST Vol 3, Issue 1, Jan-March 2012

[10] Sudeep D. Thepade, Yogita D. Shinde, "Robust CBIR using sectorisation of hybrid wavelet transforms with Cosine-Walsh, Cosine-Kekre, Cosine-Hartley combinations", International Conference on Pervasive Computing (ICPC), January 8-9, 2015, India.

[11] Swati Agarwal, A. K. Verma, Nitin Dixit, "Content based Image retrieval using color edge detection and discrete wavelet transform ", IEEE conference on issues and challenges in intelligent computing techniques, 2014.

[12] http://wang.ist.psu.edu/docs/related/Image.orig. 